\title{
The Design of Multi Degree of Freedom Manipulator Controller Based on 485 Bus
}

\author{
Bin Jiao and Tongqing Feng \\ Electrical School, Shanghai Dianji University, Shanghai, China
}

\begin{abstract}
According to the requirement of high efficiency, convenience and accuracy for the robot, this paper presents a system of multi degree of freedom manipulator based on 12 Series MCU. Through the man-machine interface to control the robot joint motion of the motor, the panel shows the actual position of the current joint as well as the current status of the motor and other information, and the whole device can realize the linkage of 6 joint motor. The experiment shows the design of multi degree of freedom manipulator has high reliability, strong anti-interference ability, high efficiency and simple, low price, and it's easy to realize the communication of point to point, point to many.
\end{abstract}

Keywords—485 bus; SCM; multi degree of freedom manipulator

\section{INTRODUCTION}

As one of the best tools to replace human labor, robots will be the best assistants of human beings from the date of its birth to the present and the future, and are widely used in industrial production and agricultural applications. Robot joint control ${ }^{[1]}$ is the core part of the robot system. Every complete robot system is composed of a single robot joint driver. Manipulator is a combination of mechanical technology, electronic technology and automatic control technology. It is an important branch of robotics, and has broad application prospects. As a highly potential advanced robot technology, robot joint control is widely used in special working environment such as military industry, nuclear industry, space and deep sea exploration, and plays an important role in the fields of biomedicine, minimally invasive surgery and underground mining. The robot joint controller is the core of the control system. Its performance directly determines the stability and reliability of the system.

Robot joints control has been developed in the United States, Britain, Singapore, France, Australia and other countries. For example, the concept model of Toshiba's trial home robot is equipped with a newly developed open robot driver and controller using distributed object technology, so it is easy to add new functions. The company also established an open robot driver architecture using decentralized object technology, and developed other controllers based on the architecture. The IRC5 driver with modular design is the fifth generation robot joint controller launched by ABB company in the near future. Its birth marks a major innovation and progress in the field of robot control technology. The research on robots in China started in the early 1970s. In the period of "85", "95" and "15", the National 863 plan has formulated a series of corresponding high-tech research and development plans ${ }^{[2]}$. Through the start of "75", "85" and "95", our country has basically mastered the core technology of robot manufacturing and design technology, control and driving system design technology, and robot software and programming.

The development of multi degree of freedom robot hand has greatly expanded the workspace of traditional robot, and its application field is more and more widely. Its important development direction is to realize full autonomy ${ }^{[3]}$. Due to the limitation of the conditions, this paper only completes the control and design of every joint driver of the robot. That is the part of the red box in Figure I.

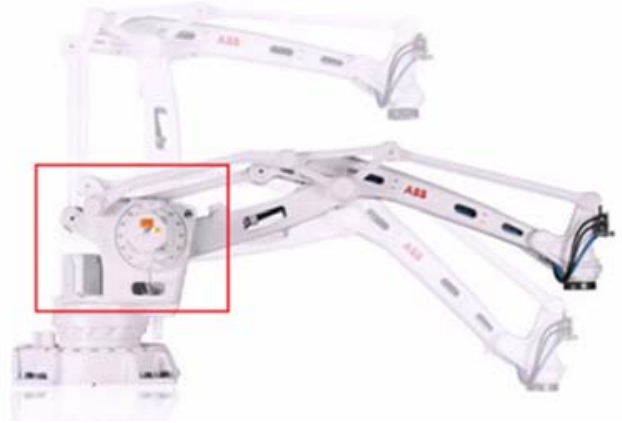

FIGURE I. INDUSTRIAL MINITYPE MANIPULATOR

\section{DESIGN SCHEME}

The robot intelligent joint driver of the multi degree of freedom manipulator control system is based on 12 series single chip microcomputer and LM629 high precision motion controller, and uses 485 Fieldbus to communicate with other equipment[4]. The whole system includes upper computer, slave computer and 485 bus part. The master block diagram of the overall design is shown in Figure II below.

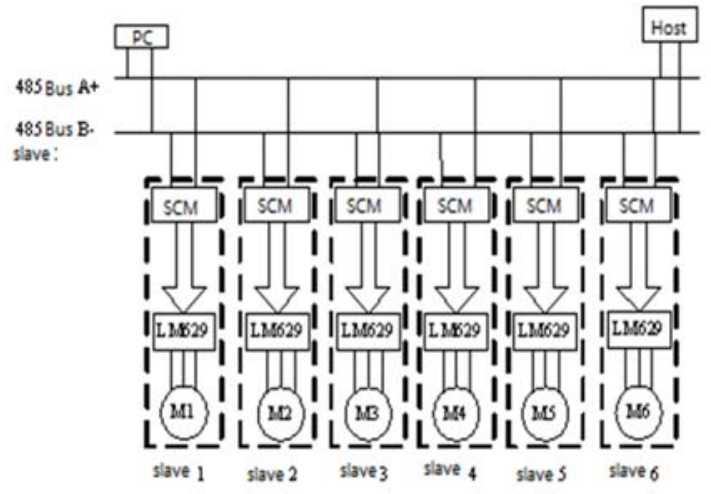

FIGURE II. THE OVERALL STRUCTURE DIAGRAM OF CONTROL SYSTEM 


\section{A. LM629 Application}

The system describes a servo motor system composed of LM629 ${ }^{[5]}$. The main processor can communicate with LM629 through I/O port, thus reducing the difficulty of configuring trapezoidal speed curve and digital filter compensator. An incremental encoder provides feedback for closed loop position servo system; trapezoidal velocity curve generator calculates the necessary trajectory for the position or velocity mode in operation. In operation, LM629 subtracts the actual position (feedback position) with the set position (the position of the curve generator), and the position error is driven by the digital filter to drive the motor to the set position. The structure diagram of the typical LM629 system is shown in Figure III below.

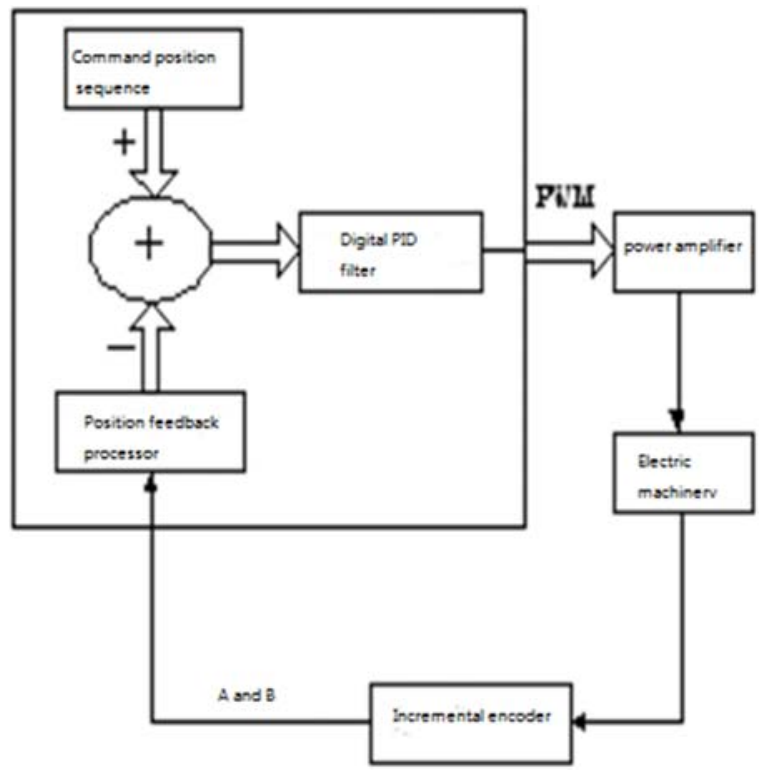

FIGURE III. LM629 TYPICAL SYSTEM STRUCTURE BLOCK DIAGRAM

\section{B. The Velocity Distribution Generator}

The trapezoidal velocity distribution generator is used to calculate the desired position of the motor's time axis. In the position operation mode, the main processor specifies the maximum speed, the final position and the acceleration, and the LM629 uses this information to reach the maximum speed through a specific acceleration value or must start decelerating to stop to the final position of the set. Under the speed operation mode, the motor accelerates to the set speed according to the set acceleration value and maintains the setting speed until the instruction requests it to stop. The speed is maintained by the desired position in advance. If there is interference in motion, the overall average speed will remain unchanged.

The desired trajectory parameters need to be calculated as follows: for example, a 100 line rotary encoder expects the motor to have an acceleration of $1 \mathrm{r} / \mathrm{s}$ before reaching 600rpm, and then decelerates to stop at the exact position of the 100 rotation at the distance starting position, then the number of trajectories can be calculated as follows ${ }^{[6]}$ :

Order: $\mathrm{P}=$ target location (encoder value)

$\mathrm{R}=$ encoder line number $\times 4$ (system resolution)

$$
=100 \times 4=400
$$

The result: $\mathrm{P}=400 \times$ resolution

$=400 \times 100$ rotation

$=40000$ count value (the value to be loaded)

$=00009 \mathrm{C} 40$ (written to the sixteen hexadecimal number of LM629)

Order: V= speed (count / sampling period)

$\mathrm{T}=$ sampling time $=341 \mathrm{us}$ (using $6 \mathrm{MHz}$ clock)

$\mathrm{C}=$ conversion factor $=1 / 60$

Then: $\mathrm{V}=\mathrm{R} \times \mathrm{T} \times \mathrm{C} \times$ expected speed value

$=400 \times 341 \mathrm{E}-6 \times 1 / 60 \times 600$ to $=1.364$

$=1.364 \times 65536=89391.104$ (approximate value)

$=89391$ (the reserved value to be loaded)

$=00015 \mathrm{D} 2 \mathrm{~F}$ (written to the sixteen hexadecimal number of LM629)

Order: $\mathrm{A}=$ acceleration (count value / sampling period / sampling period).

$=\mathrm{V} /$ sampling period $=\mathrm{R} \times \mathrm{T} \times \mathrm{C} \times$ expected speed $/$ sampling period.

$=\mathrm{R} \times \mathrm{T} \times \mathrm{T} \times$ expected acceleration (turn $/ \mathrm{sec} / \mathrm{sec}$ )

$=400 \times 341 \mathrm{E}-6 \times 341 \mathrm{E}-6 \times 1 \mathrm{turn} / \mathrm{sec} / \mathrm{sec}$

$=4.66 \mathrm{E}-5=4.66 \mathrm{E}-5 \times 65536$

$=3.048$ (approximate value)

$=3$ (the reserved value to be loaded)

$=00000003$ (written to the sixteen hexadecimal number of LM629)

The interface circuit of LM629 high precision motion control chip is shown in Figure IV below.

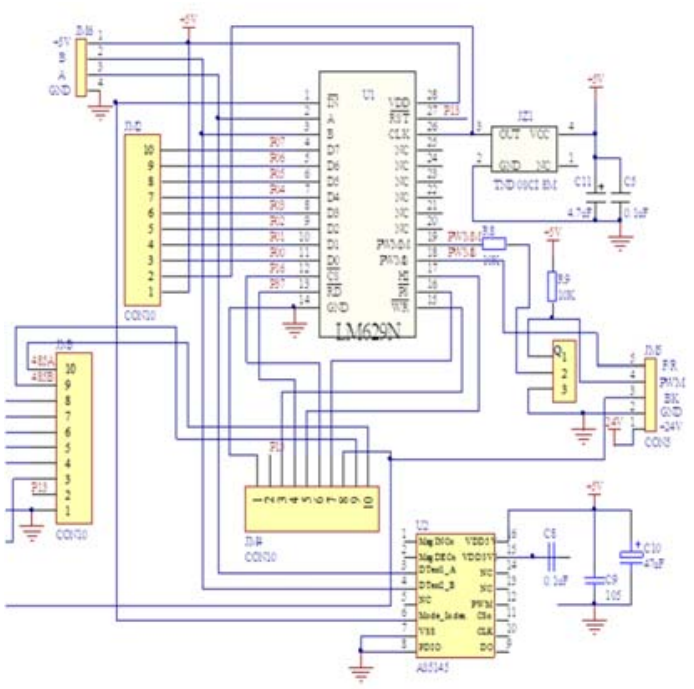

FIGURE IV. THE TERFACE CIRCUIT OF LM629 CHIP 


\section{THE SOFTWARE DESIGN}

The operation process of the VB interface operation interface $^{[7]}$ is shown in Figure V (A), (B), (C), (D), (E): the system that enters the VB man-machine interface, chooses to click the function to click in, and then hits the first step, and enters the first step interface. Input the required motion parameters (rotation angle, speed and acceleration). After opening the serial port, click start. Then the instructions are passed to the slave machine through the 485 bus, and then the data is processed through the lower machine, so that the corresponding motor can complete a series of operation. After waiting for the motor to complete the specified action, click Save again, and store all the parameters before input into the Excel form. After saving data, the interface will go back to the interface of Figure V (b). Click on the second step, enter the second step to try the interface, repeat the last operation, and complete the second step comparison. Such a cycle of operation 10 times, completed a set of actions of all tests, and each step of the trial and data are saved to the Excel table

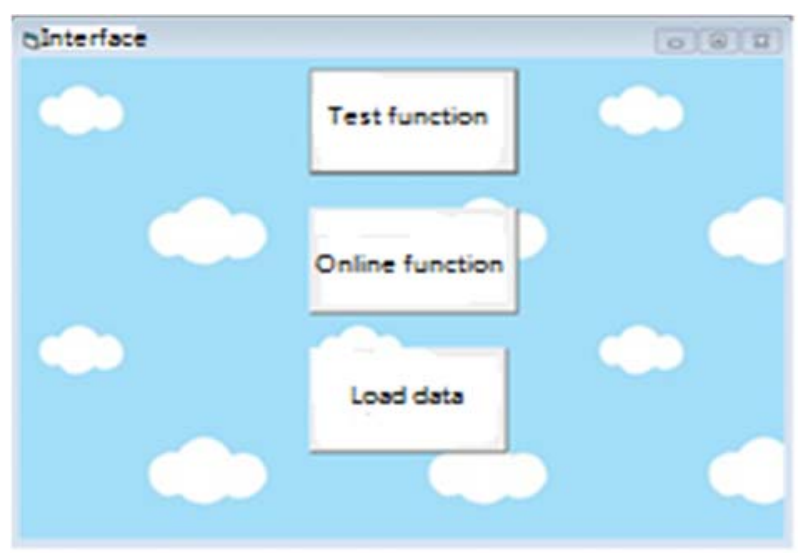

FIGURE V (A). SELECTING TEST COMPARISON FUNCTION

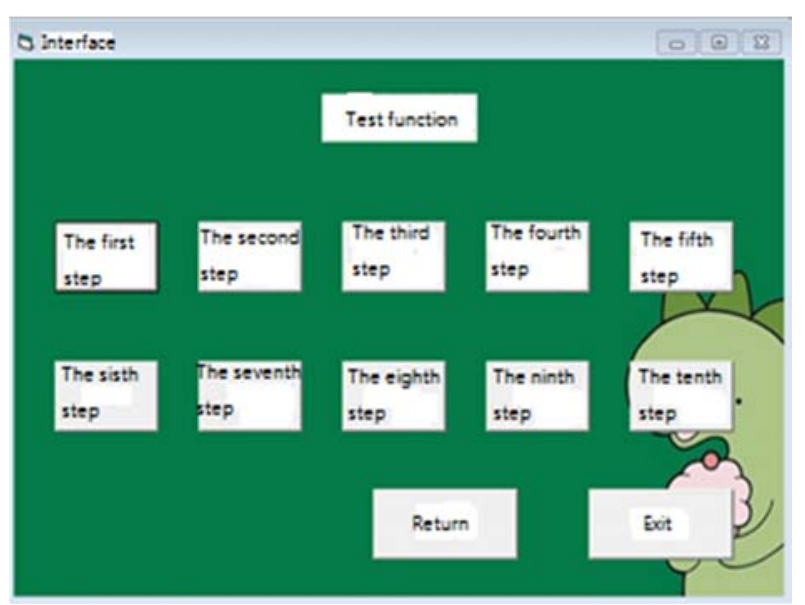

FIGURE V (B). ENTERING THE FIRST STEP

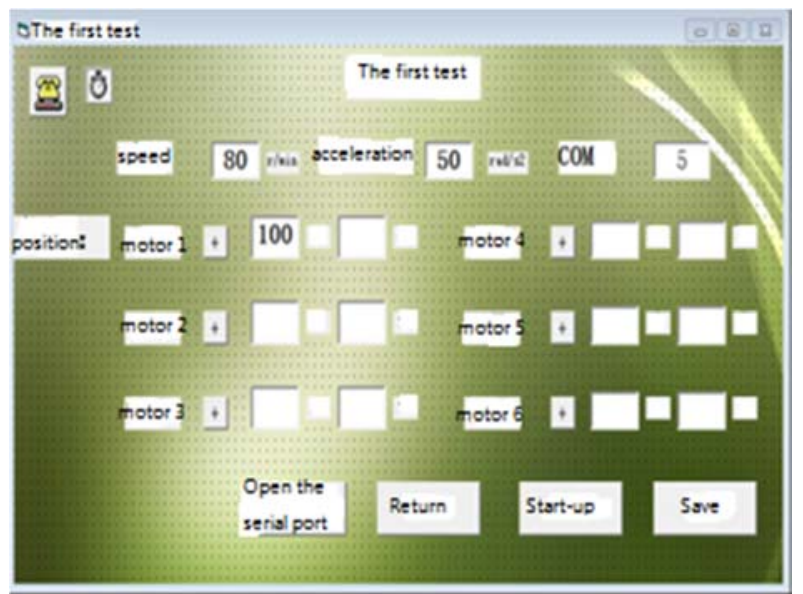

FIGURE V (C).STARTING THE FIRST STEPS SAVE DATA AFTER MOTION PARAMETERS

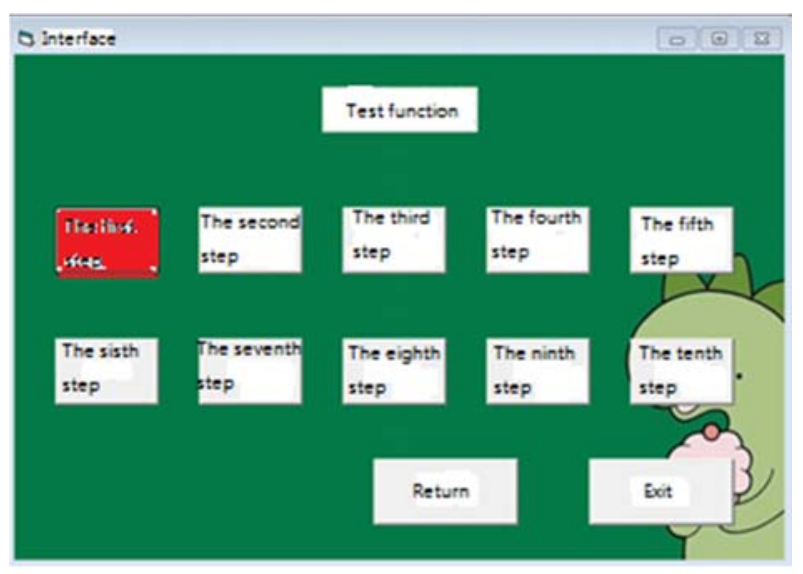

FIGURE V (D). ENTERING THE SECOND STEP

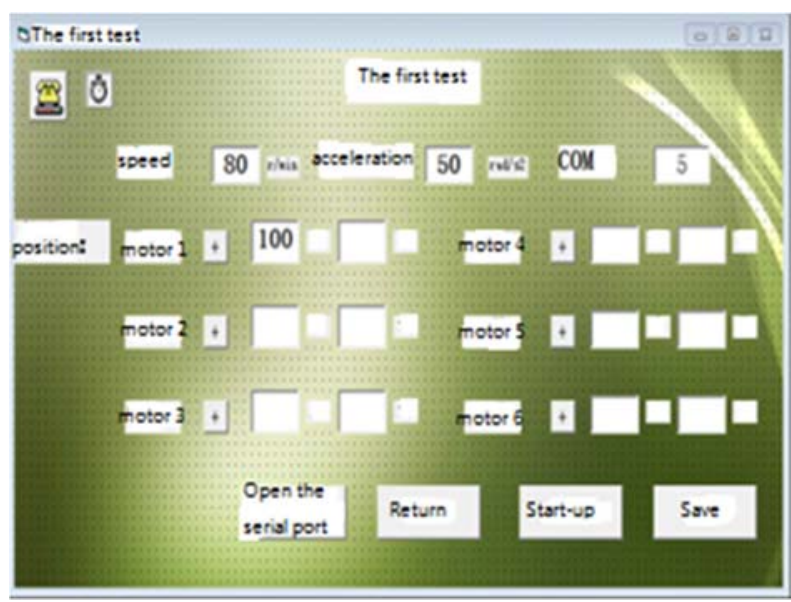

FIGURE V (E). STARTING THE SECOND STEPS AND SAVE DATA AFTER COMPARING ALL MOTION PARAMETERS

\section{SUMMARY}

The design of multi degree of freedom manipulator is very typical, and other more complex and powerful operation processing system is also based on this aera ${ }^{[8]}$. After testing, it is found that the multi degree of freedom manipulator based on the 
485 bus has stable performance and strong anti-interference ability, and the cost of development and production is relatively low. It can achieve good results in practical application, and can achieve the desired goal and have good promotion and utilization value.

\section{REFERENCES}

[1] C. Xuedong Zhou , M. hao Jia W chuan. Research on motion control system of modular multi legged walking robot [J]. China Mechanical Engineering, 10, (2016).

[2] D. Ming Jun, L. Hua Meng. Seven DOF data arm multi axis servo motion controller design of [J]. mechanical design, 11, (2015).

[3] G. You Xin, a full translation of Wang Yi. DC motor century application technology. Science and Technology Press, 2016:133 148

[4] W. Rui fang, X. Fang. Design and research of motion controller for lapping and polishing robot. [J]. microcomputer information, 9-2:261-263, (2017)

[5] W. Dong, L. Yu, W. Liang will apply. Protel DXP 2004 for 100 cases. Electronic Industry Press, (2011)

[6] D. I. Voltages.Across an Area of a Network [J]. Power Systems, IEEE Transactions on, 27(2):993, (2012)

[7] M. Young, The Technical Writer's Handbook. Mill Valley, CA: University Science, 1989.

[8] Fernando Lopez-Lopez, orial Gomis - Bellmunt. A novel educational platform to teach CAN open field bus[J]. Computer Applications in Engineering Education, 19(2):377, (2011). 